Annuaire suisse de politique de développement

13 | 1994

Annuaire Suisse - Tiers Monde 1994

\title{
Les ressources humaines dans le développement des services de santé
}

Eric Burnier et Nicolaus Lorenz

\section{(2) OpenEdition}

1 Journals

Édition électronique

URL : http://journals.openedition.org/aspd/1059

DOI : 10.4000/aspd.1059

ISSN : 1663-9669

Éditeur

Institut de hautes études internationales et du développement

Édition imprimée

Date de publication : 1 janvier 1994

Pagination : 206-215

ISSN : 1660-5934

\section{Référence électronique}

Eric Burnier et Nicolaus Lorenz, «Les ressources humaines dans le développement des services de santé », Annuaire suisse de politique de développement [En ligne], 13| 1994, mis en ligne le 19 décembre 2012, consulté le 08 septembre 2020. URL : http://journals.openedition.org/aspd/1059 ; DOI : https:// doi.org/10.4000/aspd.1059

Ce document a été généré automatiquement le 8 septembre 2020

(c) The Graduate Institute I Geneva 


\title{
Les ressources humaines dans le développement des services de santé
}

\author{
Eric Burnier et Nicolaus Lorenz
}

1 "A quelques exceptions près, tous les pays se heurtent aux mêmes problèmes fondamentaux pour ce qui est des ressources humaines dans le secteur de la santé : Il n'y a pas assez de dispensateurs de soins primaires et il y a trop de spécialistes; le personnel de santé est concentré dans les zones urbaines; la formation en santé publique, politique de santé et gestion sanitaire a été relativement négligée; la formation médicale est subventionnée, alors que les médecins peuvent avoir des revenus élevés et que beaucoup travaillent dans le secteur privé." Investir dans la santé, rapport sur le développement dans le monde 1993, Banque mondiale.(1)

\section{Introduction}

Quelle est l'attente d'une personne soudainement frappée par une maladie ou un accident? La réponse est en gros la même sous toutes les latitudes : obtenir dans les plus brefs délais l'aide la plus efficace et autant que possible doublée de réconfort. Ainsi, dans le domaine de la santé, la question des ressources humaines est-elle centrale, en quantité aussi bien qu'en qualités, ces dernières étant non seulement techniques mais également morales.

Or, avec un budget pour la santé se situant actuellement entre $3 \%$ et $6 \%$ du budget national et dont 60 à $80 \%$ sont réservés au coût du personnel, les pays en développement sont forcément très limités. Bien que le personnel de santé soit en nombre croissant dans tous les pays, beaucoup de problèmes restent à résoudre :

- Les fonctions curatives l'emportent sur les fonctions préventives, en ressources personnelles aussi bien qu'en moyens financiers. La guérison d'un patient a un effet positif immédiat sur son entourage, valorise ceux qui l'ont obtenue et entraîne plus facilement le soutien de la population qu'une campagne de prévention dont les effets sont généralement plus diffus, 
plus globaux, plus lents à se manifester et impliquent souvent pour leur réussite non seulement un apport financier mais également un changement de comportement.

- Il y a inégalité dans la répartition régionale des personnels de santé ; les médecins mais également d'autres catégories de personnels de santé, notamment les sages-femmes, se concentrent dans les zones urbaines au détriment des zones rurales ; l'équilibre entre généralistes et spécialistes, voire entre spécialistes divers, n'est pas respecté ; le manque d'anesthésistes, de psychiatres ou d'ingénieurs en assainissement est très fréquent.

- Il y a désaffection du personnel infirmier partout dans le monde, mais surtout dans le Tiers Monde. Les raisons en sont multiples : conditions de vie (horaires, salaire) difficiles, manque de perspective de carrière, migration des pays pauvres vers les pays riches, déséquilibre de l'allocation des ressources à la formation en faveur des spécialistes (médecins et dentistes) plutôt que pour les autres catégories de personnels, dégradation progressive de l'image publique (2), recrutement du personnel local le plus qualifié par les agences internationales ou les organisations non gouvernementales (3).

- On ne prend pas en compte le rôle très Important joué par le secteur non professionnel, tels les comités de santé villageois au Bénin, les pharmacies communautaires de Madagascar, etc.

Consciente de tous ces problèmes et ces dérives, l'OMS s'est préoccupée depuis $1976 \mathrm{du}$ Développement coordonné des ressources humaines et sanitaires (DCRHS) (4). Ce processus de développement recouvre trois fonctions générales: la planification des ressources humaines englobant l'élaboration d'une politique quant aux aspects qualitatifs des ressources humaines pour la santé et aux aspects quantitatifs de l'offre et des besoins; l'éducation et la formation qui ne dépendent pas uniquement du système de santé lui-même; enfin la gestion des ressources humaines: l'emploi, l'affectation et la motivation de toutes les catégories d'agents de santé, le programme de formation professionnelle continue (théorique, pratique et technique) et aussi leur difficile coordination et leur évaluation.

5 En 1978, la conférence de Alma-Ata, organisée conjointement par l'OMS et l'UNICEF, suivie un an plus tard du développement du concept de la "Santé pour tous en l'an 2000", marque une étape majeure dans l'histoire de la santé à l'échelle mondiale. La volonté d'inclure la communauté elle-même dans la réalisation des objectifs visant à la santé pour tous aboutit à la mise en place d'un nouveau système de santé, plus équitable, celui des Soins de santé primaires (SSP). Mais inévitablement cette tâche, visant davantage à la promotion de la santé qu'aux seuls soins curatifs, revient aux agents de santé, notamment aux agents de santé villageois (ASV), de catégorie très variable selon les pays. Plus agents sociaux que cliniciens, sans formation approfondie donc bon marché et par conséquent à disposition en abondance, ces ASV doivent paradoxalement exécuter des tâches très difficiles telles que la communication avec la population: le fait de vivre dans la communauté, la connaissance de la langue, de la culture locales ne sont pas toujours suffisants pour transmettre de manière pertinente des messages visant à obtenir de la population des changements de comportement tels que l'usage de latrines, d'eau propre, l'élimination des ordures, etc., ceci d'autant plus que la population est peu disposée à prêter une oreille attentive à des agents de santé formés en quelques mois, dont les capacités professionnelles n'apparaissent pas crédibles et qui de plus s'occupent de prévention plus que de soins curatifs (5).

6 Ajoutons que le rôle des ASV se complique encore par leur position charnière entre le système de santé officiel et la communauté desservie : qu'ils soit perçus comme l'ultime 
maillon du système ou comme représentants de leur communauté, ils se trouvent le plus souvent en porte-à-faux.

7 Cependant, s'ils sont bien encadrés et que leur travail fait l'objet d'une supervision régulière et positive, les ASV et les accoucheuses traditionnelles peuvent apporter une contribution non négligeable au système de soins. Ainsi par exemple, les 30 agents de santé villageois (ASV) et les 25 matrones traditionnelles du district sanitaire de Kyabé, Tchad, formés entre 1978 et 1987, ont continué d'être soutenus par le projet de la Coopération suisse dans la mesure où Ils correspondaient à une demande réelle de la communauté. Les défections ont été importantes chez les ASV, sauf lorsqu'ils dépendaient de l'aire de responsabilité d'un dispensaire. Par contre, le nombre des matrones s'est maintenu et a même augmenté, si bien que les séances de formation se sont poursuivies jusqu'à ce jour avec l'appui d'une ONG française, en donnant une place toute particulière à la référence des femmes enceintes à risques.

8 Au fil des années, la complexité des tâches relevant du secteur de la santé, cela dans un contexte de restrictions budgétaires progressives, a amené les responsables nationaux et internationaux à s'interroger sur les moyens d'optimiser les ressources disponibles. On s'est longtemps préoccupé davantage du nombre de personnel de santé à former par rapport à la population (un médecin pour 5000 habitants, un ASV pour 1000 habitants sont des objectifs régulièrement avancés) que de la qualité de cette formation et par conséquent de son coût.

9 Très variable selon les pays, le rapport médecin - infirmier(ère) diplômé(e) idéal est probablement de 1 pour 2 à 3 . Le coût de la formation d'un médecin est équivalent à celui de la formation de 3 à 4 infirmières ou de 6 à 9 aides-infirmières selon les pays. Mais la formation n'est pas tout. Ce qui n'est pas assez pris en compte ce sont les charges financières consécutives à la présence d'un médecin: non seulement son salaire mais également le coût entraîné par l'équipement nécessaire à l'exercice de sa profession ainsi que le salaire des personnels de santé dépendant de lui, etc. Or, dans la majorité des pays du globe, on continue à former des médecins et notamment des spécialistes, sans se soucier assez de l'enveloppe budgétaire permettant leur emploi futur. Dans un pays pauvre par exemple, le "coût total" d'un médecin équivaut à l'emploi de six aides-infirmières, chacunes d'entre elles pouvant travailler dans une communauté rurale. Ceci montre bien que la planification du personnel de santé est également une affaire de volonté politique.

"Le plus haut niveau d'éducation pour chaque pays est celui qui répond le mieux aux besoins locaux" (6). A cette recommandation de la 20ème conférence du CIOMS, nous ajouterons cette citation de Brian Abel-Smith: "Dans toutes les sociétés ce sont les pauvres qui ont le plus besoin de soins de santé et qui ont le moins de chance de les recevoir ; et dans beaucoup de pays, les riches pourraient se contenter de moins" (7). Pour répondre à de pareils défis, il faudrait tenir beaucoup mieux compte des besoins de la communauté en ce qui concerne la répartition des différentes catégories de personnel, la définition adéquate de leur rôle, l'analyse des tâches relevant de chaque profession ainsi que les prévisions quant aux fluctuations des besoins (8). Outre la compétence technique, on attend en effet des différents personnels d'autres qualités qui ne s'apprennent guère sur les bancs d'une école professionnelle, tels la capacité à communiquer, à enseigner, à superviser, l'esprit d'équipe, la création d'un climat d'accueil et de mise en confiance des familles et des patients, etc. 
11 On se rend également compte que le personnel de santé est le plus souvent mal formé aux tâches de gestion, d'administration et d'éducation sanitaire qui représentent une bonne part du cahier des charges. On peut se demander avec Vaughan (3) si, plutôt que d'inclure ce type de formation dans le curriculum des agents de santé, la création d'une nouvelle catégorie de professionnels de l'administration et de la gestion des services de santé, notamment à l'échelle du district, ne serait pas plus efficace.

Il faut aussi noter qu'un besoin de formation plus adapté n'est pas seulement exprimé par les autorités et les planificateurs, mais également et très fortement par les agents de santé eux-mêmes qui souhaitent autant un perfectionnement professionnel qu'une possibilité de passer à un échelon supérieur, donc d'accéder à un meilleur salaire.

Cependant, même en admettant que l'on puisse atteindre le rapport idéal du nombre des différents agents de santé par rapport à la population, à la définition et à la répartition de leurs diverses tâches, la qualité des soins, au plus large sens du terme, ne serait pas garantie pour autant. Nous voudrions ici donner le point de vue d'un antropologue, Bernard Hours (9) : "La plupart des acteurs ont été formés à exercer un métier spécifique, sans aucune attention au fait qu'à la suite de leur formation, ils seront le plus souvent incapables d'exercer leur métier (par manque de médicaments, de matériel), ce qui entraîne un certain manque de motivation. Sans être particulièrement altruiste, ce personnel aime faire du bon travail pour autant qu'il soit payé en retour par du respect et du prestige social. La connaissance est inutile tant qu'elle n'est pas reconnue dans la société par un salaire décent et des conditions de travail acceptables. Dans pareil contexte, le but n'est pas seulement de mettre le personnel devant son devoir. Il est de transformer des acteurs frustrés, négatifs, en acteurs positifs, aimant leur vie professionnelle et ses résultats. (...) Il est moins important de dresser une liste précise des tâches à accomplir, déjà bien Identifiée, que de reconnaitre clairement quels sont les obstacles actuels et quelles conditions sont nécessaires à la réalisation correcte des tâches prévues par les groupes professionnels (médecins, infirmiers, assistants médicaux, aides-médicaux ruraux, sages-femmes)."

Dans ce contexte en constante évolution, voyons maintenant la position et le rôle de la DDA dans l'appui à la formation et à l'emploi du personnel de santé dans les pays en développement.

\section{Le rôle de la DDA dans le soutien à la formation des personnels de santé}

Consciente du rôle crucial de la formation du personnel à tous les niveaux, la DDA a toujours encouragé et soutenu ce volet de la coopération, au sein de chaque programme, mais aussi par des interventions diversifiées auprès d'instituts de formations nationales, écoles, universités, etc. Il n'y a cependant à notre connaissance pas de document émanant de la DDA qui définisse une politique générale dans le domaine de la santé, si ce n'est un projet de programme de travail du service sectoriel santé, paru en 1986.

Le soutien à la formation des agents de santé par la DDA concerne toute la gamme du personnel de santé, des paramédicaux (agents de santé ruraux, assistants médicaux, sages-femmes, techniciens de laboratoire et de radiologie, personnel infirmier) aux spécialistes ou aux chercheurs, en passant par les médecins de districts. La DDA a 
également contribué à la formation de l'Ecole inter-Etats d'ingénieurs de l'équipement rural (EIER) et du Centre régional pour les technologies appropriées dans le domaine de l'eau potable et de l'assainissement (CREPA) au Burkina-Faso, par l'intermédiaire de l'Ecole polytechnique fédérale de Lausanne.

17 Par ailleurs, la DDA se soucie d'offrir une formation adaptée aux besoins, ce qui n'est de loin pas le cas avec tous les bailleurs de fonds, ONG comprises. Ainsi sont priviligiées des approches participatives fondées sur la pratique, l'analyse des tâches, donc une formation par objectifs et sujette à des évaluations répétées.

Outre ce type classique de formation, il faut aussi souligner l'importance de la formation "sur le tas" réalisée dans le cadre des programmes de santé, par exemple en Tanzanie où a été privilégiée une formation initiale courte des ASV sur place, suivie d'un accompagnement régulier de longue durée (formation-supervision continue). Un tel système coûte moins cher qu'une formation de plusieurs mois suivie de stages pratiques et a l'avantage de garder les ASV sur leur lieu de travail : c'est le formateursuperviseur qui se déplace selon un programme établi et assure le suivi.

ans de nombreux programmes de la DDA, notamment au Bénin, au Tchad, en Tanzanie, des incitations matérielles sont offertes aux personnels de santé, tels des logements (par exemple pour l'infirmier ou/et la sage-femme lors de la construction de dispensaires) ou des indemnités diverses. Par contre, une incitation sous forme de formation, ou de promesse de bourse de formation, ne s'est pas avérée une bonne formule, car elle peut fausser les rapports entre le bailleur de fonds et le personnel local (espoirs insatisfaits, concurrences, revendication). D'autre part, l'objectif de la formation doit être de combler une lacune, de répondre à un besoin au sein du système de santé et non pas de gratifier un agent méritant.

Une autre difficulté réside dans l'absence de reconnaissance officielle des compétences de tel ou tel agent de santé formé "sur le tas", par exemple dans une structure missionnaire, face à un nouveau venu bardé d'un diplôme d'Etat mais dont les connaissances sont encore très théoriques. Les conflits sont alors difficiles à résoudre, chacun revendiquant un acquis différent, et il faut trouver un compromis de cas en cas.

Voyons maintenant les diverses interventions de la DDA dans le domaine de la santé.

\section{1) Bourses de formation professionnelle}

A la section Bourses et Cours de la DDA, la santé vient en deuxième position, après l'enseignement technique et professionnel. A titre d'exemple, la section a financé en 199256 bourses de stage dans ce domaine, ce qui représente $12 \%$ de la totalité des bourses octroyées.

La répartition de ces bourses est la suivante :

\section{Bourses de stage en immunologie auprès de l'OMS}

La section finance des bourses depuis 1972 pour des ressortissants des pays en développement. Les boursiers effectuent leurs stages au Centre de recherche et de formation en immunologie de l'OMS, à Lausanne et à Genève, formation visant à l'application pratique de l'immunologie et de la biologie moléculaire au diagnostic et à la prévention des maladies infectieuses rencontrées dans leurs pays d'origine. Cette formation, qui se déroule à raison de deux stages annuels, l'un en français, l'autre en anglais, est doublée d'un suivi des anciens stagiaires par l'OMS, en coordination avec les 
programmes nationaux en santé publique. De plus, les anciens boursiers renforcent les capacités de recherche de leurs pays en collaborant à des programmes nationaux ou régionaux. En onze ans, plus de 500 stagiaires ont bénéficié des bourses de la DDA (en 1992,32 boursiers provenaient d'Afrique du Nord, d'Afrique au Sud du Sahara, d'Amérique centrale et d'Amérique du Sud, du Moyen-Orient, d'Asie du Sud et d'Extrême-Orient). De 1985 à 1994, l'ensemble du montant consacré à ce programme par la section Bourses et Cours s'est élevé à 7800000 francs. et des infirmiers venus des pays en développement se former en Suisse; ce programme est géré par la section Bourses et Cours de la DDA mais à la demande et sur financement d'autres sections. Ainsi par exemple, 9 médecins et infirmiers cancérologues ont pu suivre des stages de spécialisation dans les hôpitaux cantonaux de Genève, Bâle et StGall, pour des périodes de 3 à 6 mois. Trois bourses de 4 ans ont été également octroyées à un étudiant mozambicain et à deux médecins malgaches, à l'Institut de pharmacologie et de toxicologie de l'Université de Lausanne; à leur retour, ils seront parmi les premiers pharmacologues spécialistes de leurs pays.

Enfin, dans le cadre des programmes en régie, de nombreuses bourses d'études de durée moyenne à longue ont permis à des laborantins, des infirmiers et des médecins d'acquérir dans des pays étrangers (Afrique, Europe, Canada) une spécialisation, notamment en Santé publique. 


\section{2) Soutien à des institutions de formation dans les pays en développement}

$\mathrm{Au}$ Centre universitaire des sciences de la santé de Yaoundé, la DDA a également octroyé en 1990 un crédit unique de 200000 francs pour l'Installation d'un atelier de réparation du matériel de laboratoire ainsi que pour la formation de deux techniciens. A l'heure actuelle, cet atelier donne toute satisfaction.

La Société missionnaire Bethlehem, basée à Immensee, est active depuis très longtemps au Zimbabwe. En 1984 et 1986, la DDA a participé à ce programme par un soutien financier de 552000 francs à l'Ecole d'infirmières de Musiso afin de l'aider à former en quatre ans 16 jeunes gens et jeunes filles comme assistants médicaux, répartis ensuite dans les services de santé publics et privés du pays.

En 1984 et 1987, la DDA a octroyé un financement de 406000 francs à une ONG indienne, le Voluntary Health Assistance India (VHAI), pour le Centre de santé du Jamkhed. Ce projet associe la formation d'agents de santé villageois de sexe féminin chargés des soins de santé primaire et d'éducation sanitaire, de l'amélioration de la situation de la femme et de la participation des villages à l'amélioration des conditions de vie et de santé.

Au Népal, la DDA soutient depuis dix ans l'Ecole de formation professionnelle de Jiri, en zone rurale de montagne. Le volet santé de cette école concerne la formation d'infirmières sages-femmes auxiliaires qui obtiennent en trois ans un certificat reconnu par l'Etat. Les 12 à 15 élèves inscrites chaque année participent elles-mêmes au recyclage des accoucheuses traditionnelles. L'avantage de cette formation décentralisée par rapport à la formation dans la capitale est que $86 \%$ des élèves restent en zone rurale pour exercer leur métier alors que celles formées en zones urbaines n'en sortent plus. Toujours au Népal, la Croix-Rouge suisse, partiellement financée par la DDA, a développé un programme de formation en ophtalmologie destiné aux différentes catégories d'agents de santé, ASV, infirmiers.

La DDA participe également à des programmes de planification familiale en Indonésie à Sulawesi et à Java, en collaboration avec l'UNICEF. Elle soutient aussi le programme de stages pour jeunes médecins, organisé par Medicus Mundi Suisse, débuté en 1979.

Depuis 1970, la Coopération suisse appuie la formation de personnel paramédical au Tchad dans le cadre de son unique Ecole nationale de santé publique et de service social (ENSPSS); des infirmiers et sages-femmes diplômés d'Etat, ainsi que des assistants sociaux et, par intermittence, des laborantins sont formés dans cette Ecole. Outre des bourses (100 par an, soit un tiers du nombre total d'étudiants), la DDA met des enseignants à la disposition de l'Ecole. A partir de 1986, elle contribué à réviser le curriculum de formation, puis elle a pris en charge une des sections, celle des sagesfemmes. Les principales contraintes rencontrées ont été le surnombre d'étudiants, faute de sélection appropriée à l'entrée, l'impossibilité de faire respecter les contrôles d'apprentissage lors des examens et les défaillances administratives globales de l'ENSPSS. En 1990, le programme, géré en régie pour l'Institut tropical suisse (ITS), a réorienté sa composante formation vers un appui direct à la Division de la planification et de la formation du Ministère de la Santé publique du Tchad. Par ailleurs, il a assuré un appui à l'élaboration des programmes d'enseignement de la Faculté des sciences de 
la santé et des Instructions diagnostiques et thérapeutiques à l'usage du personnel paramédical tchadien.

Au Mali, l'Ecole d'Infirmiers du 1 er Cycle (EIPC) de Sikasso a été construite en 1987, dans le cadre du Programme Santé Mali-Suisse, géré par l'IUED pour la DDA. L'objectif principal de cette Ecole est de contribuer au développement du personnel sociosanitaire par la formation initiale des infirmiers et infirmières du 1 er Cycle, le recyclage du personnel socio-sanitaire de la région de Sikasso, et, accessoirement, la recherche appliquée dans le domaine de la formation du personnel infirmier. L'objectif secondaire est de décentraliser l'enseignement, jusqu'alors donné uniquement dans la capitale. Plus de 100 élèves fréquentent cette Ecole; un diplôme d'Infirmier du 1 er Cycle reconnu par l'Etat leur est attribué après trois ans de formation.

En Tanzanie, à Ifakara, un soutien logistique au Médical Assistant Training Center et un enseignement pratique et théorique donné par les coopérants du Programme de l'ITS ainsi que par les médecins envoyés par l'ONG Solidarmed, qui bénéficie de l'appui financier DDA, sont une composante importante au développement des ressources humaines de ce pays. Selon la volonté du gouvernement tanzanlen, cette Ecole, de même que les huit autres que compte le pays, est située en pleine zone rurale, de manière à ce que les étudiants restent en contact permanent avec le milieu dans lequel ils auront, une fois diplômés, à travailler. Après avoir formé dans un premier temps des agents ruraux de santé, l'école est devenue un centre de formation d'assistants médicaux qui bénéficient pour l'enseignement clinique de la présence de l'Hôpital Saint-Francis dont tous les médecins et plusieurs infirmiers sont également enseignants. Chaque année une volée de 30 à 40 assistants médicaux reçoivent leur diplôme d'Etat qui leur permet de travailler soit dans des hôpitaux soit dans des Centres de santé. Il s'agit donc d'une formation non universitaire, essentiellement pratique.

41 Inclusion d'un volet formation dans les projets

42 Que ce soit dans les programmes en régie ou dans d'autres programmes ou projets administrés par des œuvres missionnaires ou des ONG dans le domaine de la santé, le volet formation et développement des ressources humaines a toujours une place importante. Sur une liste de 16 ONG suisses travaillant dans ce domaine, 15 mentionnent des activités de formation, sous toutes sortes de formes: séminaires, ateliers, participations à des cours de longue durée, bourses, formation continue, etc.

\section{3) Participation indirecte aux cours de formation}

43 Par son appui financier, notamment à l'ITS et à l'IUED, la DDA soutient également des cours de formation professionnelle de ces deux instituts.

Depuis 1950, l'Institut tropical suisse a délivré annuellement un cours de médecine et santé publique tropicale et préparé plus de 800 médecins, vétérinaires, biologistes, infirmières, sages-femmes et laborantines à travailler dans les pays en développement. Ce cours a considérablement évolué dans sa forme et son approche pédagogique au cours des années afin de tenir compte des besoins, inclure les aspects de gestion et s'articuler en modules distincts.

45 A l'Institut universitaire d'études du développement, un séminaire sur l'approche pluridisciplinaire de la santé est suivi chaque année par des étudiants venus de tous les 
continents. Certains d'entre eux reçoivent également des mandats de courte durée pour étudier sur le terrain et en collaboration avec les coopérants de l'IUED certaines questions qui se posent, notamment en santé publique urbaine. L'intégration entre programmes de développement de la santé régis par l'IUED et enseignement académique trouve là sa réalisation.

Enfin, les deux instituts ont monté en 1989,1990 et 1991, des ateliers conjoints d'une semaine de formation pratique sur un thème de santé publique pour les coopérants de leurs programmes et leurs partenaires.

\section{Conclusion}

47 Ces différents exemples montrent l'intérêt que la Coopération suisse manifeste pour une formation diversifiée de l'ensemble des ressources humaines dans le cadre de la santé. Nous aimerions ici souligner le danger qu'entrâne la multiplicité des cours, séminaires, ateliers, etc., organisés par toutes les ONG et agences de coopération engagées dans les pays en développement. Chacun pense bien faire, selon sa propre expérience, mais il y a un risque d'éparpillement des possibilités, voire des tentations pour le personnel de santé dont le besoin réel de perfectionnement professionnel peut être occulté par les avantages temporaires, notamment financiers (per diem), accompagnant souvent ce perfectionnement.

Dans l'intérêt même du Ministère de la Santé des pays concernés, la coordination de ces apports divers à la formation devrait être une priorité, en fonction des besoins en personnel des diverses catégories mais aussi des moyens à disposition, pour ensuite assurer les salaires et le fonctionnement. Il est également nécessaire de redéfinir les curriculums d'études, pour donner à l'ensemble du système une homogénéité nationale.

C'est nous semble-t-il par l'intégration à un plan d'enseignement prévu au niveau national de chaque pays où elle Intervient que la coopération étrangère aurait le plus de chance de rendre des services à long terme. En ce sens le soutien de la DDA aux institutions nationales, universités, écoles professionnelles ou instituts s'inscrit depuis longtemps dans cette perspective.

Enseigner est une chose, évaluer les résultats à court et surtout à long terme de l'impact de cet enseignement en est une autre. Si certains rapports et documents font, à titre individuel, mention de telles évaluations, une vue d'ensemble des résultats obtenus par la DDA dans le domaine de la formation du personnel sanitaire, fait à ce jour encore défaut. UUED UUED 


\section{AUTEURS}

\section{ERIC BURNIER}

Chargé de cours à I' IUED

\section{NICOLAUS LORENZ}

Wissenschaftlicher Mitarbeiter, Schweizerisches tropeninstitut, Socinstrasse 57, 4051 Basel 